and sexual contacts of hepatitis C. There are epidemiological studies to support these findings.

\section{P59 A SYSTEMATIC REVIEW OF ASSOCIATIONS BETWEEN SUBSTANCE USE AND SEXUAL RISK BEHAVIOUR, STIS AND UNPLANNED PREGNANCY IN WOMEN}

${ }^{1,2}$ Natalie Edelman* ${ }^{3}$ Richard De Visser, ${ }^{4}$ Catherine Mercer, ${ }^{1}$ Jackie Cassell. ${ }^{1}$ Brighton and Sussex Medical School, Brighton and Hove, UK; ${ }^{2}$ University of Brighton, Brighton and Hove, UK; ${ }^{3}$ University of Sussex, Brighton and Hove, UK; ${ }^{4}$ University College London, London, UK

\subsection{6/sextrans-2015-052126.102}

Background/introduction Associations between substance use and sexual risk among general populations of women may be helpful in the development of a sexual risk assessment tool for community health settings.

Aim(s)/objectives To review the evidence for whether smoking, alcohol and drug use variables are associated with reporting of unprotected sexual intercourse, multiple partnerships, STI diagnoses and unplanned pregnancy in women aged 16-44 years.

Methods Seven electronic databases were searched for probability population surveys published between 31/1/1994 and 31/1/ 2014 that reported on at least one of the outcomes above. Studies were included on women aged 16-44 years in the European Union, Australia, New Zealand, USA or Canada. An independent reviewer screened $10 \%$ of title and abstract exclusions and all full-text papers.

Results Three papers were identified. Current smoking was associated with unplanned pregnancy in the last year (Wellings 2013) and with current non-use of contraception among women (Xaverius 2009). Reporting ever smoking daily was also associated with reporting larger numbers of lifetime sexual partners (Cavazos-Rehg, 2011). Drug use in the last year (excepting cannabis) was associated with unplanned pregnancy (Wellings 2013). Cavazos-Rehg, 2011 found a dose response between lifetime partner numbers and heaviness of marijuana and alcohol use. Conversely Xaverius, 2009 found alcohol use was lower among those reporting current non-use of contraception.

Discussion/conclusion No clear direction emerged for the association with alcohol use, in contrast to drug use and smoking. Further research is needed to establish if alcohol has utility in a women's sexual risk assessment tool for community use.

\section{P60 ASSOCIATIONS BETWEEN SUBSTANCE USE AND SEXUAL RISK BEHAVIOUR AMONG WOMEN AGED 16-44 YEARS: EVIDENCE FROM BRITAIN'S THIRD NATIONAL SURVEY OF SEXUAL ATTITUDES AND LIFESTYLES (NATSAL-3)}

\begin{abstract}
1,2 Natalie Edelman*, ${ }^{3}$ Philip Prah, ${ }^{1}$ Jackie Cassell, ${ }^{4}$ Richard de Visser, ${ }^{3}$ Catherine Mercer. ${ }^{1}$ Brighton and Sussex Medical School, Brighton and Hove, UK; ${ }^{2}$ University of Brighton, Brighton and Hove, UK; ${ }^{3}$ University College London, London, UK; ${ }^{4}$ University of Sussex,
\end{abstract} Brighton and Hove, UK

10.1136/sextrans-2015-052126.103

Background/introduction Taking account of substance use may be important when developing a sexual risk assessment tool for use with women in community health settings.
Aim(s)/objectives To examine whether different measures of substance use have different associations with key sexual risk behaviours among women in the British general population (rather than women attending sexual health clinics who typically report higher risk behaviour).

Methods We analysed data from 4,911 female participants aged 16-44 in Natsal-3, a national probability sample survey undertaken 2010-2012, using multivariable regression to examine the associations between substance use variables and reporting: multiple $(2+)$ partners in the last year; non-use of condoms with multiple partners in the last year; non-use of condoms at first sex with most recent partner.

Results Reporting multiple partners was associated with current smoking (OR 1.59, 95\% CI 1.30-1.93), weekly binge drinking (OR 2.47, 95\% CI 1.97-3.10), and drug use ever (OR 1.45, 95\% CI 1.20-1.75). Similarly, reporting non-use of condoms with multiple partners was also associated with current smoking (OR 1.39, 95\% CI 1.09-1.78), weekly binge drinking (OR 2.47, 95\% CI 1.90-3.21) and drug use ever (OR 1.48, 95\% CI 1.171.88). Non-use of condoms at first sex with most recent partner was only associated with current smoking (OR 1.47 95\% CI 1.25-1.73) and weekly binge drinking (OR 1.41 95\% CI 1.14-1.73).

Discussion/conclusion Differences were found to exist in how substance use variables are associated with the sexual risk behaviours studied. Different substance use questions may therefore be useful in identifying and distinguishing different sexual risk behaviours profiles in community settings.

\section{P61 PSYCHOSOCIAL DETERMINANTS OF HIV DISCLOSURE TO CONFIDANTS WITH DIFFERENT HIV STATUS}

${ }^{1}$ Aliia Makhamatova* ${ }^{2}$ Alena Suvorova, ${ }^{3}$ Andrey Belyakov, ${ }^{4}$ Andrey Ustinov, ${ }^{5}$ Olga Levina, ${ }^{6}$ Robert Heimer. ${ }^{1}$ Saint Petersburg State University, Saint Petersburg, Russia; ${ }^{2}$ Saint Petersburg Institute for Informatics and Automation of RAS, Saint Petersburg, Russia; ${ }^{3}$ Petersburg Center for Control of AIDS and Infectious Diseases, Saint Petersburg, Russia; ${ }^{4}$ Bekhterev's Scientific Research Institute of Psychiatry, Saint Petersburg, Russia; ${ }^{5}$ NGO Stellit, Saint Petersburg, Russia; ${ }^{6}$ Yale University, New Haven, USA

\subsection{6/sextrans-2015-052126.104}

Background/introduction Informing HIV-negative people by people living with HIV (PLWH) about their status might probably have great HIV preventive effect. That is why it's important to study the factors of HIV disclosure to confidents with different HIV status.

Aim(s)/objectives The goal of the study was to reveal the psychosocial determinants of HIV disclosure to confidents with different HIV status.

Methods In 2013 we surveyed 418 PLWH in Saint Petersburg, Russia. We employed Internalised AIDS-Related Stigma Scale (IA-RSS), SF-36 Health Status Survey, Multidimensional Scale of Perceived Social Support (MPSS), the Lubben Social Network Scale (LSNS). The interview guide also contained the question about HIV status of those people, who were informed about respondents' HIV status by respondents themselves.

Results The sample was $58 \%$ of male (mean age $=34.3$ years). An average time of identification of HIV was 6.3 years before the study. Logistic regression model explaining HIV disclosure to people with positive or/and negative HIV status included the 